\title{
Mellin Transform of an Exponential Fourier Transform Expressed in Terms of the Lerch Function
}

\author{
Robert Reynolds*, Allan Stauffer \\ Department of Mathematics and Statistics, Faculty of Science, York University, Canada
}

Cite This Paper in the following Citation Styles

(a): [1] Robert Reynolds, Allan Stauffer, "Mellin Transform of an Exponential Fourier Transform Expressed in Terms of the Lerch Function," Mathematics and Statistics, Vol.9, No.5, pp. 724-735, 2021. DOI: 10.13189/ms.2021.090512

(b): Robert Reynolds, Allan Stauffer, (2021). Mellin Transform of an Exponential Fourier Transform Expressed in Terms of the Lerch Function. Mathematics and Statistics, 9(5), 724-735. DOI: 10.13189/ms.2021.090512

Copyright @2021 by authors, all rights reserved. Authors agree that this article remains permanently open access under the terms of the Creative Commons Attribution License 4.0 International License

\begin{abstract}
The aim of this paper is to provide a table of definite integrals which includes both known and new integrals. This work is important because we provide a formal derivation for integrals in [7] not currently present in literature along with new integrals. By deriving new integrals we hope to expand the current list of integral formulae which could assist in research where applicable. The authors apply their contour integral method [9] to an integral in [8] to achieve this new integral formulae in terms of the Lerch function. In this present work, the authors provide a formal derivation for an interesting Exponential Fourier transform and express it in terms of the Lerch function. The Exponential Fourier transform has many real world applications namely, in the field of Electrical engineering, in the work of electrical transients by [10] and in the field of Civil engineering, in the work of stress analysis of boundary load on soil by [11]. The definite integral we derived in this work is given by

$$
\int_{-\infty}^{\infty} \frac{e^{-i m x}(\log (a)-i x)^{k}}{\sinh (\alpha)+\sinh (x)} d x
$$

where the variables $k, a, \alpha \in \mathbb{C},-1<\operatorname{Im}(m)<1, \alpha \neq 0$. This formal derivation is then used to derive the correct version of a definite integral transform along with new formulae. Some of the results in this work are new.
\end{abstract}

Keywords Hilbert Transform, Mellin Transform, Exponential Fourier Transform, Hyperbolic Function, Catalan's Constant

\section{Introduction}

This work provides a formal derivation for an integral in [8], the correct version for a definite integral in [4] and some new definite integrals in terms of special functions and fundamental constants. This work also looks to expand the Table 2.4.15 in [8] where similar and relevant formula are listed. In our case the constants in the formulas are general complex numbers subject to the restrictions given below. The derivations follow the method used by us in [9]. The generalized Cauchy's integral formula is given by

$$
\frac{y^{k}}{k !}=\frac{1}{2 \pi i} \int_{C} \frac{e^{w y}}{w^{k+1}} d y .
$$

where $C$ is in general an open contour in the complex plane where the bilinear concomitant has the same value at the end points of the contour. This method involves using a form of equation (2) then multiplies both sides by a function, then takes a definite integral of both sides. This yields a definite integral in terms of a contour integral. Then we multiply both sides of equation (2) by another function and take the infinite sum of both sides such that the contour integral of both equations are the same. 


\section{Derivations of the contour integral representations}

In this section we derive the definite integral and Lerch representation of the contour integral.

\subsection{Definite integral of the contour integral}

We use the method in [9]. The cut and contour are in the first quadrant of the complex $m+w$-plane. The cut approaches the origin from the interior of the first quadrant and the contour goes round the origin with zero radius and is on opposite sides of the cut. Using a generalization of Cauchy's integral formula we first replace $y \rightarrow-i x+\log (a)$ followed by multiplying both sides by $\frac{e^{-i m x}}{\sinh (\alpha)+\sinh (x)}$ then taking the definite integral with respect $x \in(-\infty, \infty)$ to get

$$
\begin{aligned}
& \int_{-\infty}^{\infty} \frac{e^{-i m x}(\log (a)-i x)^{k}}{k !(\sinh (\alpha)+\sinh (x))} d x=\frac{1}{2 \pi i} \int_{-\infty}^{\infty} \int_{C} \frac{a^{w} w^{-k-1} e^{-i x(m+w)}}{\sinh (\alpha)+\sinh (x)} d w d x \\
& =\frac{1}{2 \pi i} \int_{C} \int_{-\infty}^{\infty} \frac{a^{w} w^{-k-1} e^{-i x(m+w)}}{\sinh (\alpha)+\sinh (x)} d x d w \\
& =-\frac{1}{2 \pi i} \int_{C} i \pi a^{w} \operatorname{sech}(\alpha) w^{-k-1} \operatorname{csch}(\pi(m+w))\left(-e^{-i \alpha(m+w)}\right. \\
& \left.+e^{i \alpha(m+w)} \cosh (\pi(m+w))\right) d w \\
& =\frac{1}{2 \pi i} \int_{C} \pi a^{w} \operatorname{sech}(\alpha) w^{-k-1} \tanh \left(\frac{1}{2} \pi(m+w)\right)\left(\operatorname{coth}^{2}\left(\frac{1}{2} \pi(m+w)\right) \sin (\alpha(m+w))\right. \\
& -i \cos (\alpha(m+w))) d w
\end{aligned}
$$

from equation (2.4.14.9) in [8] where $\operatorname{Re}(m+w)>0$ and $\operatorname{Re}(\alpha)>0$.

\subsection{The Lerch function}

The Lerch function has a series representation given by

$$
\Phi(z, s, v)=\sum_{n=0}^{\infty}(v+n)^{-s} z^{n}
$$

where $|z|<1, v \neq 0,-1, .$. and is continued analytically by its integral representation given by

$$
\Phi(z, s, v)=\frac{1}{\Gamma(s)} \int_{0}^{\infty} \frac{t^{s-1} e^{-v t}}{1-z e^{-t}} d t=\frac{1}{\Gamma(s)} \int_{0}^{\infty} \frac{t^{s-1} e^{-(v-1) t}}{e^{t}-z} d t
$$

where $\operatorname{Re}(v)>0$, and either $|z| \leq 1, z \neq 1, \operatorname{Re}(s)>0$, or $z=1, \operatorname{Re}(s)>1$.

\subsection{Infinite sum of the contour integral}

\subsubsection{Derivation of the first contour integral}

In this section we will derive the first contour integral given by

$$
\frac{1}{2 \pi i} \int_{C} \pi a^{w} \operatorname{sech}(\alpha) w^{-k-1} \operatorname{coth}\left(\frac{1}{2} \pi(m+w)\right) \sin (\alpha(m+w)) d w
$$

Using equation (2) we form two equations by first replacing $y \rightarrow y+i \alpha$ then multiplying both sides by $e^{i \alpha m}$ and for the second equation we replace $\alpha \rightarrow-\alpha$ and take their difference to get

$$
-\frac{i e^{-i \alpha m}\left(e^{2 i \alpha m}(y+i \alpha)^{k}-(y-i \alpha)^{k}\right)}{2 k !}=\frac{1}{2 \pi i} \int_{C} w^{-k-1} e^{w y} \sin (\alpha(m+w)) d w
$$

Next we replace $y \rightarrow \log (a)+\frac{2 \pi(y+1)}{2}$ and multiply by $-2 \pi \operatorname{sech}(\alpha) e^{\frac{2 \pi m(y+1)}{2}}$ and take the infinite sum over $y \in[0, \infty)$ and simplify in terms of the Lerch function to get 


$$
\begin{aligned}
& -\frac{i \pi^{k+1} \operatorname{sech}(\alpha) e^{\pi m-i \alpha m} \Phi\left(e^{m \pi},-k, \frac{-2 i \alpha+2 \log (a)+2 \pi}{2 \pi}\right)}{k !}+\frac{i \pi^{k+1} \operatorname{sech}(\alpha) e^{\pi m+i \alpha m} \Phi\left(e^{m \pi},-k, \frac{2 i \alpha+2 \log (a)+2 \pi}{2 \pi}\right)}{k !} \\
& +\frac{i \pi \operatorname{sech}(\alpha) e^{-i \alpha m}\left(e^{2 i \alpha m}(\log (a)+i \alpha)^{k}-(\log (a)-i \alpha)^{k}\right)}{2 k !} \\
& \quad=-\frac{1}{2 \pi i} \sum_{y=0}^{\infty} \int_{C} 2 \pi a^{w} \operatorname{sech}(\alpha) w^{-k-1} e^{\pi(y+1)(m+w)} \sin (\alpha(m+w)) d w-\frac{1}{2 \pi i} \int_{C} \pi a^{w} \operatorname{sech}(\alpha) w^{-k-1} \sin (\alpha(m+w)) d w \\
& \quad=-\frac{1}{2 \pi i} \int_{C} \sum_{y=0}^{\infty} 2 \pi a^{w} \operatorname{sech}(\alpha) w^{-k-1} e^{\pi(y+1)(m+w)} \sin (\alpha(m+w)) d w-\frac{1}{2 \pi i} \int_{C} \pi a^{w} \operatorname{sech}(\alpha) w^{-k-1} \sin (\alpha(m+w)) d w \\
& \quad=\frac{1}{2 \pi i} \int_{C} \pi a^{w} \operatorname{sech}(\alpha) w^{-k-1} \operatorname{coth}\left(\frac{1}{2} \pi(m+w)\right) \sin (\alpha(m+w)) d w
\end{aligned}
$$

from equation (1.232.1) in [7] where $\operatorname{Im}(m+w)>0$ in order for the sum to converge.

\subsubsection{Derivation of the second contour integral}

In this section we will derive the second contour integral given by

$$
-\frac{1}{2 \pi i} \int_{C} i \pi a^{w} \operatorname{sech}(\alpha) w^{-k-1} \tanh \left(\frac{1}{2} \pi(m+w)\right) \cos (\alpha(m+w)) d w
$$

Using equation (2) we form two equations by first replacing $y \rightarrow y+i \alpha$ then multiplying both sides by $e^{i \alpha m}$ and for the second equation we replace $\alpha \rightarrow-\alpha$ and add both to get

$$
\frac{e^{-i \alpha m}\left(e^{2 i \alpha m}(y+i \alpha)^{k}+(y-i \alpha)^{k}\right)}{k !}=\frac{1}{2 \pi i} \int_{C} 2 w^{-k-1} e^{w y} \cos (\alpha(m+w)) d w
$$

Next we replace $y \rightarrow \log (a)+\frac{2 \pi(y+1)}{2}$ and multiply by $-i \pi(-1)^{y} \operatorname{sech}(\alpha) e^{\pi m(y+1)}$ and take the infinite sum over $y \in[0, \infty)$ and simplify in terms of the Lerch function to get

$$
\begin{aligned}
- & \frac{i \pi^{k+1} \operatorname{sech}(\alpha) e^{\pi m-i \alpha m} \Phi\left(-e^{m \pi},-k, \frac{-2 i \alpha+2 \log (a)+2 \pi}{2 \pi}\right)}{k !}-\frac{i \pi^{k+1} \operatorname{sech}(\alpha) e^{\pi m+i \alpha m} \Phi\left(-e^{m \pi},-k, \frac{2 i \alpha+2 \log (a)+2 \pi}{2 \pi}\right)}{k !} \\
+ & \frac{i \pi \operatorname{sech}(\alpha) e^{-i \alpha m}\left((\log (a)-i \alpha)^{k}+e^{2 i \alpha m}(\log (a)+i \alpha)^{k}\right)}{2 k !} \\
= & -\frac{1}{2 \pi i} \sum_{y=0}^{\infty} \int_{C} 2 i \pi(-1)^{2 y} a^{w} \operatorname{sech}(\alpha) w^{-k-1} e^{\frac{2 \pi(y+1)(2 m+w)}{t}} \cos (\alpha(m+w)) \\
& +\frac{1}{2 \pi i} \int_{C} \pi a^{w} \operatorname{sech}(\alpha) w^{-k-1} \cos (\alpha(m+w)) d w \\
= & -\frac{1}{2 \pi i} \int_{C} \sum_{y=0}^{\infty} 2 i \pi(-1)^{2 y} a^{w} \operatorname{sech}(\alpha) w^{-k-1} e^{\frac{2 \pi(y+1)(2 m+w)}{t}} \cos (\alpha(m+w)) \\
& +\frac{1}{2 \pi i} \int_{C} \pi a^{w} \operatorname{sech}(\alpha) w^{-k-1} \cos (\alpha(m+w)) d w \\
= & -\frac{1}{2 \pi i} \int_{C} i \pi a^{w} \operatorname{sech}(\alpha) w^{-k-1} \tanh \left(\frac{1}{2} \pi(m+w)\right) \cos (\alpha(m+w)) d w
\end{aligned}
$$

from equation (1.232.1) in [7] where $\operatorname{Im}(m+w)>0$ in order for the sum to converge. 


\section{Exponential Fourier transform in terms of the Lerch function}

Theorem 3.1. For $k, a, \alpha \in \mathbb{C}$ and $\frac{1}{2}<\operatorname{Im}(m)<1$,

$$
\begin{aligned}
\int_{-\infty}^{\infty} \frac{e^{-i m x}(\log (a)-i x)^{k}}{\sinh (\alpha)+\sinh (x)} d x & \\
=i \pi & \operatorname{sech}(\alpha) e^{-i \alpha m}\left(e^{2 i \alpha m}(\log (a)+i \alpha)^{k}\right. \\
& -\pi^{k} e^{\pi m}\left(\Phi\left(-e^{m \pi},-k, \frac{-i \alpha+\log (a)+\pi}{\pi}\right)+\Phi\left(e^{m \pi},-k, \frac{-i \alpha+\log (a)+\pi}{\pi}\right)\right. \\
& \left.\left.+e^{2 i \alpha m}\left(\Phi\left(-e^{m \pi},-k, \frac{i \alpha+\log (a)+\pi}{\pi}\right)-\Phi\left(e^{m \pi},-k, \frac{i \alpha+\log (a)+\pi}{\pi}\right)\right)\right)\right)
\end{aligned}
$$

Proof. Since the right-hand side of equation (3) is equal to the sum of equations (8) and (11) we can equate the left-hand sides and simplify the factorial term to get the quoted result. See section (3.1) in [4].

\section{Derivations of definite integrals}

In this section we provide formal derivations for definite integrals known and new. Some of these results are present in the books of Gradshteyn and Ryzhik [7], Prudnikov et al. [8] and Bierens De Haan [3]. In some cases we also provide errata.

\subsection{Derivation of entry 2.4.14.9 in [8]}

\section{Proposition 4.1.}

$$
\begin{aligned}
& \int_{-\infty}^{\infty} \frac{e^{-i m x}}{\sinh (\alpha)+\sinh (x)} d x \\
&=\pi \operatorname{sech}(\alpha) \tanh \left(\frac{\pi m}{2}\right)\left(\operatorname{coth}^{2}\left(\frac{\pi m}{2}\right) \sin (\alpha m)-i \cos (\alpha m)\right)
\end{aligned}
$$

Proof. Use equation (12) and set $k=0$ and simplify using entry (2) in Table below (64:12:7) in [1]. Note the result in equation (3.2.30) in [4] is in error.

\subsection{Derivation of entry 2.4.14.10 in [8]}

\section{Proposition 4.2.}

$\int_{-\infty}^{\infty} \frac{x e^{-i m x}}{\sinh (\alpha)+\sinh (x)} d x=\pi \operatorname{sech}(\alpha)\left(i e^{i \alpha m}\left(\alpha \operatorname{coth}(\pi m)+i \pi \operatorname{csch}^{2}(\pi m)\right)+e^{-i \alpha m} \operatorname{csch}(\pi m)(\pi \operatorname{coth}(\pi m)+i \alpha)\right)$

Proof. Use equation (12) and set $k=1$ and simplify using entry (1) in Table below (64:12:7) in [1].

\subsection{Derivation of entry 2.4.14.11 in [8]}

Theorem 4.3. For $k, \alpha \in \mathbb{C}$ and $\frac{1}{2}<\operatorname{Re}(m)<1$,

$$
\begin{aligned}
\int_{-\infty}^{\infty} \frac{x^{k} e^{m x}}{\sinh (\alpha)+\sinh (x)} d x= & i \pi \operatorname{sech}(\alpha) e^{-\alpha m+\frac{i \pi k}{2}}\left((i \alpha)^{k}-\pi^{k} e^{i \pi m}\left(\Phi\left(-e^{i m \pi},-k, \frac{i \alpha}{\pi}+1\right)\right.\right. \\
& \left.\left.+e^{2 \alpha m}\left(\Phi\left(-e^{i m \pi},-k, 1-\frac{i \alpha}{\pi}\right)+\Phi\left(e^{i m \pi},-k, 1-\frac{i \alpha}{\pi}\right)\right)-\Phi\left(e^{i m \pi},-k, \frac{i \alpha}{\pi}+1\right)\right)\right)
\end{aligned}
$$

Proof. Use equation (12) and set $a=1, m=m i$ and simplify. 


\subsection{Derivation of entry 2.4.14.12 in [8]}

\section{Proposition 4.4.}

$$
\begin{aligned}
& \int_{-\infty}^{\infty} \frac{e^{2 x / 3} x^{2}}{(\sinh (x)+\sinh (4 \pi))^{2}} d x \\
& =\frac{1}{27} \pi^{2} \operatorname{sech}^{3}(4 \pi)\left(\pi \left(3(27 \sqrt{3}-8)\left(5 \sinh \left(\frac{4 \pi}{3}\right)+\sinh \left(\frac{20 \pi}{3}\right)\right)\right.\right. \\
& \left.\quad-5(72+25 \sqrt{3}) \cosh \left(\frac{4 \pi}{3}\right)+(72+25 \sqrt{3}) \cosh \left(\frac{20 \pi}{3}\right)\right) \\
& \left.-36 \cosh (4 \pi)\left((3+2 \sqrt{3}) \sinh \left(\frac{8 \pi}{3}\right)+(6 \sqrt{3}-1) \cosh \left(\frac{8 \pi}{3}\right)\right)\right)
\end{aligned}
$$

Proof. Use equation (4.3) and take the first partial derivative with respect to $\alpha$ and simplify. Next set $k=2, m=2 / 3, \alpha=4 \pi$ and simplify using entry (4) in Table below (64:12:7) in [1].

\subsection{Derivation of entry 2.4.14.13 in [8]}

\section{Proposition 4.5.}

$$
\int_{-\infty}^{\infty} \frac{e^{2 x / 3} x}{\sinh (x)+\sinh (3 \pi)} d x=\frac{2 e^{\pi}\left(4-3 \sqrt{3}+(2+6 \sqrt{3}) e^{4 \pi}\right) \pi^{2}}{3\left(1+e^{6 \pi}\right)}
$$

Proof. Use equation (4.3) and set $k=1, m=2 / 3, \alpha=3 \pi$ and simplify using entry (4) in Table below (64:12:7) in [1]. and

\section{Proposition 4.6.}

$$
\int_{-\infty}^{\infty} \frac{e^{3 x / 4} x^{2}}{\sinh (x)+\sinh (3 \pi)} d x=e^{-9 \pi / 4}\left(1+18 \sqrt{2} e^{9 \pi / 2}\right) \pi^{3} \operatorname{sech}(3 \pi)
$$

Proof. Use equation (4.3) and set $k=1, m=3 / 4, \alpha=3 \pi$ and simplify using entry (4) in Table below (64:12:7) in [1].

\subsection{Derivation of entry 2.4.14.14 in [8]}

$$
\int_{-\infty}^{\infty} x^{k} e^{-i m x} \operatorname{csch}(x) d x=2 i(-i)^{-k} \pi^{k+1} \mathbf{L i}_{-k}\left(-e^{m \pi}\right)
$$

Proof. Use equation (12) and set $\alpha=0, a=1$ and simplify using entry (4) in Table below (64:12:7) in [1].

and

$$
\int_{-\infty}^{\infty} \frac{\operatorname{csch}(x)\left(e^{-i p x}-e^{-i m x}\right)}{\sqrt{x}} d x=(-1-i) \sqrt{2 \pi}\left(\operatorname{Li}_{\frac{1}{2}}\left(-e^{m \pi}\right)-\operatorname{Li}_{\frac{1}{2}}\left(-e^{p \pi}\right)\right)
$$

Proof. Use equation (19) and form a second equation by replacing $m \rightarrow p$ and take their difference and set $k=1 / 2$.

\subsection{Derivation of entry 2.4.14.15 in [8]}

Theorem 4.7. For $\alpha \in \mathbb{C}, \frac{1}{2}<\operatorname{Re}(m)<1, \frac{1}{2}<\operatorname{Re}(p)<1$

$$
\begin{array}{r}
\int_{-\infty}^{\infty} \frac{e^{p x}-e^{m x}}{x(\sinh (\alpha)+\sinh (x))} d x=\frac{\operatorname{sech}(\alpha)}{\alpha}\left(\alpha e ^ { ( \alpha + i \pi ) m } \left(\Phi\left(-e^{i m \pi}, 1,1-\frac{i \alpha}{\pi}\right)+\Phi\left(e^{i m \pi}, 1,1-\frac{i \alpha}{\pi}\right)\right.\right. \\
\left.+e^{-2 \alpha m}\left(\Phi\left(-e^{i m \pi}, 1, \frac{i \alpha}{\pi}+1\right)-\Phi\left(e^{i m \pi}, 1, \frac{i \alpha}{\pi}+1\right)\right)\right) \\
-\alpha e^{(\alpha+i \pi) p\left(\Phi\left(-e^{i p \pi}, 1,1-\frac{i \alpha}{\pi}\right)+\Phi\left(e^{i p \pi}, 1,1-\frac{i \alpha}{\pi}\right)\right.} \\
\left.\left.+e^{-2 \alpha p}\left(\Phi\left(-e^{i p \pi}, 1, \frac{i \alpha}{\pi}+1\right)-\Phi\left(e^{i p \pi}, 1, \frac{i \alpha}{\pi}+1\right)\right)\right)+i \pi e^{\alpha(-m)}-i \pi e^{\alpha(-p)}\right)
\end{array}
$$

Proof. Use equation (12) and create a second equation by replacing $m \rightarrow p$ and take their difference. Next set $k=-1, a=$ $1, m=m i, p=\pi$ and simplify. 


\subsection{Derivation of entry 2.4.14.16 in [8]}

\section{Proposition 4.8.}

$$
\begin{aligned}
& \int_{-\infty}^{\infty} \frac{e^{4 x / 5}-e^{5 x / 6}}{x^{2}(\sinh (x)+\sinh (7 \pi))} d x \\
& \quad=-\frac{i e^{-343 \pi / 30}}{49 \pi^{3}}\left(-49(-1)^{\frac{4}{5}-\frac{511 i}{30}} \pi^{2} \Phi\left(-e^{\frac{4 i \pi}{5}}, 2,1-7 i\right)\right. \\
& \quad-49(-1)^{\frac{4}{5}-\frac{35 i}{6}} \pi^{2} \Phi\left(-e^{\frac{4 i \pi}{5}}, 2,1+7 i\right)-49(-1)^{\frac{4}{5}-\frac{511 i}{30}} \pi^{2} \Phi\left(e^{\frac{4 i \pi}{5}}, 2,1-7 i\right) \\
& +49(-1)^{\frac{4}{5}-\frac{35 i}{6}} \pi^{2} \Phi\left(e^{\frac{4 i \pi}{5}}, 2,1+7 i\right)+49(-1)^{\frac{5}{6}-\frac{259 i}{15}} \pi^{2} \Phi\left(-e^{\frac{5 i \pi}{6}}, 2,1-7 i\right) \\
& +49(-1)^{\frac{5}{6}-\frac{28 i}{5}} \pi^{2} \Phi\left(-e^{\frac{5 i \pi}{6}}, 2,1+7 i\right)+49(-1)^{\frac{5}{6}-\frac{259 i}{15}} \pi^{2} \Phi\left(e^{\frac{5 i \pi}{6}}, 2,1-7 i\right) \\
& \left.-49(-1)^{\frac{5}{6}-\frac{28 i}{5}} \pi^{2} \Phi\left(e^{\frac{5 i \pi}{6}}, 2,1+7 i\right)+e^{28 \pi / 5} \pi^{2}-e^{35 \pi / 6} \pi^{2}\right) \operatorname{sech}(7 \pi)
\end{aligned}
$$

Proof. Use equation (12) and set $a=e^{a i}, m=m i$ and simplify to get

$$
\begin{array}{r}
\int_{-\infty}^{\infty} \frac{e^{m x}(x-a)^{k}}{\sinh (\alpha)+\sinh (x)} d x \\
=i \pi \operatorname{sech}(\alpha) e^{-\alpha m+\frac{i \pi k}{2}}\left((i(a+\alpha))^{k}-\pi^{k} e^{i \pi m}\left(\Phi\left(-e^{i m \pi},-k, \frac{i a+i \alpha+\pi}{\pi}\right)\right.\right. \\
+e^{2 \alpha m}\left(\Phi\left(-e^{i m \pi},-k, \frac{i a-i \alpha+\pi}{\pi}\right)+\Phi\left(e^{i m \pi},-k, \frac{i a-i \alpha+\pi}{\pi}\right)\right) \\
\left.\left.-\Phi\left(e^{i m \pi},-k, \frac{i a+i \alpha+\pi}{\pi}\right)\right)\right)
\end{array}
$$

Next set $k=-1$ and form a second equation by replacing $a \rightarrow-a$ and take their difference. Next we form another equation by replacing $m \rightarrow p$ and take their difference. Next apply L'Hopital's rule to the right-hand side as $a \rightarrow 0$ and set $\alpha=7 \pi, m=$ $4 / 5, p=5 / 6$ and simplify.

\subsection{Derivation of entry 2.4.14.17 in [8]}

Theorem 4.9. For $a, \alpha \in \mathbb{C}$ and $\frac{1}{2}<\operatorname{Im}(m)<1$,

$$
\begin{aligned}
\int_{-\infty}^{\infty} \frac{\sqrt{x-a} e^{m x}}{\sinh (\alpha)+\sinh (x)} d x & \\
& =(-1)^{3 / 4} \pi \operatorname{sech}(\alpha) e^{\alpha(-m)}\left(\sqrt{i(a+\alpha)}-\sqrt{\pi} e^{i \pi m}\left(\Phi\left(-e^{i m \pi},-\frac{1}{2}, \frac{i(a+\alpha)}{\pi}+1\right)\right.\right. \\
& +e^{2 \alpha m}\left(\Phi\left(-e^{i m \pi},-\frac{1}{2}, \frac{i a-i \alpha+\pi}{\pi}\right)+\Phi\left(e^{i m \pi},-\frac{1}{2}, \frac{i a-i \alpha+\pi}{\pi}\right)\right) \\
& \left.\left.-\Phi\left(e^{i m \pi},-\frac{1}{2}, \frac{i(a+\alpha)}{\pi}+1\right)\right)\right)
\end{aligned}
$$

Proof. Use (23) and set $k=1 / 2$.

\subsection{Derivation of entry 2.4.14.18 in [8]}

Proposition 4.10.

$$
\int_{-\infty}^{\infty} \frac{e^{x / 2}(x-1)^{2}}{\sinh (x)+\sinh (5 \pi)} d x=e^{-5 \pi / 2} \pi\left(e^{5 \pi}(1+2 \pi(13 \pi-5))-2 \pi(1+5 \pi)\right) \operatorname{sech}(5 \pi)
$$

Proof. Use (23) and set $k=2, m=1 / 2, a=1, \alpha=5 \pi$ and simplify using entry (4) below Table in (64:12:7) in [1]. 


\subsection{Derivation of entry 2.4.14.19 in [8]}

\section{Proposition 4.11.}

$$
\begin{aligned}
& \int_{-\infty}^{\infty} e^{2 x / 3}\left(e^{x / 12}-1\right) \log (x-1) \operatorname{csch}(x) d x \\
& \quad=\frac{\left(1+(-1)^{2 / 3}\right)^{5} \pi}{2\left(1+(-1)^{3 / 4}\right)}\left(( 1 + i ) \left((-2 \sqrt{2}+(2+2 i)) \Phi^{\prime}\left(-(-1)^{2 / 3}, 0, \frac{\pi+i}{\pi}\right)\right.\right. \\
& \left.+((1+i)-i \sqrt{2})(\sqrt{3}-i) \Phi^{\prime}\left(-(-1)^{3 / 4}, 0, \frac{\pi+i}{\pi}\right)\right)+\left(1+2(-1)^{3 / 4}-i \sqrt{3}\right) \pi \\
& +(-2 i+4 \sqrt[4]{-1}-2 \sqrt{3}) \log (\pi))
\end{aligned}
$$

Proof. Use (23) and form a second equation by replacing $m \rightarrow p$ and take their difference. Next we take the first partial derivative with respect to $k$ and set $k=\alpha=0, a=1, m=2 / 3, p=3 / 4$ and simplify. Note there exists a singularity at $x=1$.

\subsection{Derivation of entry 2.4.14.20 in [8]}

\section{Proposition 4.12.}

$$
\begin{aligned}
& \int_{-\infty}^{\infty} e^{2 x / 3}\left(e^{x / 12}-1\right) \log (x) \operatorname{csch}(x) d x \\
& \quad=\frac{1}{2} \pi\left(-4 i\left(L i_{0}^{\prime}\left(0,-(-1)^{3 / 4}\right)-L i_{0}^{\prime}\left(\frac{1}{2}-\frac{i \sqrt{3}}{2}\right)\right)\right. \\
& +i(1+\sqrt{2}-\sqrt{3}) \pi+2(1+\sqrt{2}-\sqrt{3}) \log (\pi))
\end{aligned}
$$

Proof. Use (23) and form a second equation by replacing $m \rightarrow p$ and take their difference. Next we take the first partial derivative with respect to $k$ and set $k=\alpha=0=a=0, m=2 / 3, p=3 / 4$ and simplify in terms of the derivative of the polylogarithm function using entry (4) in Table below (64:12:7) in [1].

\subsection{Derivation of entry 2.4.14.21 in [8]}

\section{Proposition 4.13.}

$$
\begin{aligned}
& \int_{-\infty}^{\infty} \frac{\left(e^{2 x / 3}-e^{3 x / 4}\right) \operatorname{csch}(x)}{(x+i \pi)^{2}} d x \\
& =-\frac{(-1)^{3 / 4}}{96 \pi}\left(\pi^{2}+48\left(i C+\sqrt[12]{-1} L i_{2}(-\sqrt[3]{-1})\right.\right. \\
& +2\left(\sqrt[12]{-1} L i_{2}\left(-(-1)^{2 / 3}\right)-\sqrt[12]{-1} L i_{2}\left((-1)^{2 / 3}\right)\right. \\
& \left.\left.\left.-L i_{2}\left(-(-1)^{3 / 4}\right)+L i_{2}\left((-1)^{3 / 4}\right)\right)\right)\right)
\end{aligned}
$$

Proof. Use (23) and form a second equation by replacing $m \rightarrow p$ and take their difference then set $k=-2, m=3 / 4, p=$ $2 / 3, \alpha=\pi i, a=-\pi i$ and simplify in terms of the polylogarithm function and Catalan's constant using entry (4) in Table below $(64: 12: 7)$ in [1] and section (1.7.6) in [6].

\subsection{Derivation of entry 3.527.12 in [7]}

Proposition 4.14.

$$
\int_{-\infty}^{\infty} x^{2} \operatorname{csch}^{2}(x) d x=\frac{\pi^{2}}{3}
$$

Proof. Use (23) and set $k=2, a=0$ and simplify using entry (4) in Table below (64:12:7) in [1]. Then apply L'Hopital's rule to the right-hand as $m \rightarrow 0$. Nest take the first partial derivative with respect to $\alpha$ and set $\alpha=0$. 


\subsection{Derivation of new entry 3.527 .17 in [7]}

Proposition 4.15.

$$
\int_{-\infty}^{\infty} x^{4} \operatorname{csch}^{2}(x) d x=\frac{\pi^{4}}{15}
$$

Proof. Use (23) and set $k=4, a=0$ and simplify using entry (4) in Table below (64:12:7) in [1]. Then apply L'Hopital's rule to the right-hand as $m \rightarrow 0$ to get

$$
\int_{-\infty}^{\infty} \frac{x^{4}}{\sinh (\alpha)+\sinh (x)} d x=-\frac{1}{15} \alpha\left(-6 \alpha^{4}+10 \pi^{2} \alpha^{2}+\pi^{4}\right) \operatorname{sech}(\alpha)
$$

Next take the first partial derivative with respect to $\alpha$ and set $\alpha=0$.

\subsection{Derivation of new entry 3.527.18 in [7]}

Proposition 4.16.

$$
\int_{-\infty}^{\infty} x^{4} \operatorname{csch}^{4}(x) d x=-\frac{2}{45} \pi^{2}\left(\pi^{2}-15\right)
$$

Proof. Use (31) and take the second partial derivative with respect to $\alpha$ simplify then set $\alpha \rightarrow 0$.

\subsection{Derivation of new entry 3.527.19 in [7]}

Proposition 4.17.

$$
\int_{-\infty}^{\infty} x^{6} \operatorname{csch}^{2}(x) d x=\frac{\pi^{6}}{21}
$$

Proof. Use (23) and set $k=6, a=0$ and simplify using entry (4) in Table below (64:12:7) in [1]. Then apply L'Hopital's rule to the right-hand as $m \rightarrow 0$ to get

$$
\int_{-\infty}^{\infty} \frac{x^{6}}{\sinh (\alpha)+\sinh (x)} d x=-\frac{1}{21} \alpha\left(-6 \alpha^{6}+21 \pi^{2} \alpha^{4}+7 \pi^{4} \alpha^{2}+\pi^{6}\right) \operatorname{sech}(\alpha)
$$

Next take the first partial derivative with respect to $\alpha$ and set $\alpha=0$.

\subsection{Derivation of entry new 3.527.20 in [7]}

Proposition 4.18. For $k \in \mathbb{C}$,

$$
\int_{-\infty}^{\infty} x^{k} \operatorname{csch}(x) d x=2 i\left(2^{k+1}-1\right) e^{\frac{i \pi k}{2}} \pi^{k+1} \zeta(-k)
$$

Proof. Use (23) and set $a=m=0$ and simplify using entry (2) in Table below (64:12:7) in [1].

\subsection{Derivation of new entry 3.527.21 in [7]}

Proposition 4.19. For $k \in \mathbb{C}$,

$$
\int_{-\infty}^{\infty} e^{2 x / 3} x^{k} \operatorname{csch}^{2}(x) d x=\frac{1}{3} e^{\frac{i \pi k}{2}}\left(6 k \pi^{k} L i_{1-k}\left((-1)^{2 / 3}\right)+4 i \pi^{k+1} L i_{-k}\left((-1)^{2 / 3}\right)\right)
$$

Proof. Use (23) and set $a=0, m=2 / 3$ then take the first partial derivative with respect to $\alpha$ followed by setting $\alpha=0$ and simplify using entry (4) in Table below (64:12:7) in [1].

\subsection{Derivation of entry $B I(101)(4)$ in [3]}

Proposition 4.20.

$$
\int_{-\infty}^{\infty} x\left(1-e^{p x}\right) \operatorname{csch}(x) d x=-\frac{1}{2} \pi^{2} \tan ^{2}\left(\frac{\pi p}{2}\right)
$$

Proof. Use equation 23 and set $k=\alpha=0$ to get

$$
\int_{-\infty}^{\infty} e^{m x} \operatorname{csch}(x) d x=\pi\left(\tan \left(\frac{\pi m}{2}\right)-i\right)
$$

Next form a second equation by replacing $m \rightarrow p$. Next take the first partial derivative with respect to $m$ and $p$ of each respectively. Next take their difference and set $m=0$. 


\subsection{Derivation of new entry 3.527 .22 in [7]}

Proposition 4.21.

$$
\int_{-\infty}^{\infty} x^{3} \operatorname{csch}^{3}(x) d x=-\frac{1}{8} \pi^{2}\left(\pi^{2}-12\right)
$$

Proof. Use equation (23) and set $k=3, m=0$ then take the second partial derivative with respect to $\alpha$. Next apply L'Hopital's rule to the right-hand side as $\alpha \rightarrow 0$ and simplify. Note this process is quite lengthy.

\subsection{Derivation of new entry 3.527.23 in [7]}

Proposition 4.22.

$$
\int_{-\infty}^{\infty} x^{4} \operatorname{csch}^{4}(x) d x=-\frac{2}{45} \pi^{2}\left(\pi^{2}-15\right)
$$

Proof. Use equation (23) and set $k=4, m=0$ then take the third partial derivative with respect to $\alpha$. Next apply L'Hopital's rule to the right-hand side as $\alpha \rightarrow 0$ and simplify. Note this process is quite lengthy.

\subsection{Derivation of new entry 3.527.24 in [7]}

Proposition 4.23.

$$
\int_{-\infty}^{\infty} e^{u x} \operatorname{csch}(b x) \sinh (c x) d x=\frac{\pi \sin \left(\frac{\pi c}{b}\right)}{b \cos \left(\frac{\pi c}{b}\right)+b \cos \left(\frac{\pi u}{b}\right)}
$$

Proof. Use equation (23) and set $k=0$. Next replace $x \rightarrow b x$ and simplify. Next replace $u \rightarrow u+c$ and form a second equation by replacing $c \rightarrow-c$ and take their difference and set $\alpha=0$. Set $c=u$ and $u \rightarrow-u$ to get equation (3.541.3) in [7].

\subsection{Derivation of new entry 3.527.25 in [7]}

Proposition 4.24. For $\operatorname{Re}(c)>\operatorname{Re}(b),-1<\operatorname{Re}(u)<1$,

$$
\int_{-\infty}^{\infty} x e^{u x} \operatorname{csch}(b x) \cosh (c x) d x=\frac{\pi^{2}\left(\cos \left(\frac{\pi c}{b}\right) \cos \left(\frac{\pi u}{b}\right)+1\right)}{b^{2}\left(\cos \left(\frac{\pi c}{b}\right)+\cos \left(\frac{\pi u}{b}\right)\right)^{2}}
$$

Proof. Use equation (41) and take the first partial derivative with respect to $c$ and simplify.

\subsection{Derivation of entry 3.543.1 in [7]}

Proposition 4.25. For $-1<\operatorname{Re}(b)<1,-1<\operatorname{Im}(b)<-1 / 2, \operatorname{Re}(\alpha)>0$,

$$
\int_{-\infty}^{\infty} \frac{e^{i b x}}{\sinh (\alpha)+\sinh (x)} d x=\pi \operatorname{sech}(\alpha) \tanh \left(\frac{\pi b}{2}\right)\left(\operatorname{coth}^{2}\left(\frac{\pi b}{2}\right) \sin (\alpha b)+i \cos (\alpha b)\right)
$$

Proof. Use (23) and set $a=0, m=i b$ and simplify. Note there exists a singularity at $x=-\alpha$ when $\alpha$ is real. The integral is slowly decaying and oscillatory.

\subsection{Derivation of entry 3.511.9 in [7]}

Proposition 4.26.

$$
\int_{-\infty}^{\infty} \operatorname{csch}^{2}(x) \sinh ^{2}(m x) d x=\frac{1}{2}(2-2 \pi m \cot (\pi m))
$$

Proof. Use (23) and set $k=a=0$ and simplify using entry (2) in Table below (64:12:7) in [1]. Next form a second equation by setting $m \rightarrow-m$ and add both equations to get

$$
\int_{-\infty}^{\infty} \frac{\cosh (m x)}{\sinh (\alpha)+\sinh (x)} d x=\pi \operatorname{sech}(\alpha) \cot \left(\frac{\pi m}{2}\right) \sinh (\alpha m)
$$

Next set $m=2 m$ and take the first partial derivative with respect to $\alpha$ and set $\alpha=0$ to get

$$
\int_{-\infty}^{\infty} \operatorname{csch}^{2}(x) \cosh (2 m x) d x=-2 \pi m \cot (\pi m)
$$

Then apply L'Hopital's rule to the right-hand side to equations (46) as $m \rightarrow 0$ and take the difference from equation (46) to get quoted result. 


\section{Hilbert transform in terms of the Lerch function}

In this section we look at the Hilbert transform see section (15.1) in [5] in terms of the Lerch function.

Theorem 5.1. For $\operatorname{Re}(a)>0, \operatorname{Re}(m)>0$,

$$
\int_{-\infty}^{\infty} \frac{\operatorname{csch}(x)(\pi a \sin (m x)+x \cos (m x))}{\pi^{2} a^{2}+x^{2}} d x=\frac{1}{a}-2 e^{\pi(-m)} \Phi\left(-e^{-m \pi}, 1, a+1\right)
$$

and

$$
\int_{-\infty}^{\infty} \frac{\operatorname{csch}(x)(x \sin (m x)-\pi a \cos (m x))}{\pi^{2} a^{2}+x^{2}} d x=0
$$

Proof. Use equation (23) and set $k=-1$ and rationalize the denominator and equate real and imaginary parts to achieve the stated result.

Theorem 5.2. For $\operatorname{Re}(a)>0, \operatorname{Re}(m)>0$,

$$
\int_{-\infty}^{\infty} \frac{\pi \operatorname{csch}(x)\left(\left(x^{2}-\pi^{2} a^{2}\right) \sin (m x)-2 \pi a x \cos (m x)\right)}{\left(\pi^{2} a^{2}+x^{2}\right)^{2}} d x=2 e^{\pi(-m)} \Phi\left(-e^{-m \pi}, 2, a+1\right)-\frac{1}{a^{2}}
$$

up

$$
\int_{-\infty}^{\infty} \frac{\pi \operatorname{csch}(x)((\pi a-x)(\pi a+x) \cos (m x)-2 \pi a x \sin (m x))}{\left(\pi^{2} a^{2}+x^{2}\right)^{2}} d x=0
$$

Proof. Use equation (47) and take the first partial derivative with respect to $a$ and simplify.

\section{Table of integrals}

In this section we summarize our results in the form of a table for easy referencing by researchers interested in these results. 


\begin{tabular}{|c|c|}
\hline$f(x)$ & $\int_{-\infty}^{\infty} f(x) d x$ \\
\hline$\frac{e^{x / 2}(x-1)^{2}}{\sinh (x)+\sinh (5 \pi)}$ & $e^{-5 \pi / 2} \pi\left(e^{5 \pi}(1+2 \pi(13 \pi-5))-2 \pi(1+5 \pi)\right) \operatorname{sech}(5 \pi)$ \\
\hline$x^{2} \operatorname{csch}^{2}(x)$ & $\frac{\pi^{2}}{3}$ \\
\hline$\frac{x^{4}}{\sinh (\alpha)+\sinh (x)}$ & $-\frac{1}{15} \alpha\left(-6 \alpha^{4}+10 \pi^{2} \alpha^{2}+\pi^{4}\right) \operatorname{sech}(\alpha)$ \\
\hline$x^{4} \operatorname{csch}^{2}(x)$ & $\frac{\pi^{4}}{15}$ \\
\hline$x^{4} \operatorname{csch}^{4}(x)$ & $-\frac{2}{45} \pi^{2}\left(\pi^{2}-15\right)$ \\
\hline$\frac{x^{6}}{\sinh (\alpha)+\sinh (x)}$ & $-\frac{1}{21} \alpha\left(-6 \alpha^{6}+21 \pi^{2} \alpha^{4}+7 \pi^{4} \alpha^{2}+\pi^{6}\right) \operatorname{sech}(\alpha)$ \\
\hline$x^{6} \operatorname{csch}^{2}(x)$ & $\frac{\pi^{6}}{21}$ \\
\hline$x^{k} \operatorname{csch}(x)$ & $2 i\left(2^{k+1}-1\right) e^{\frac{i \pi k}{2}} \pi^{k+1} \zeta(-k)$ \\
\hline$e^{2 x / 3} x^{k} \operatorname{csch}^{2}(x)$ & $\frac{1}{3} e^{\frac{i \pi k}{2}}\left(6 k \pi^{k} \operatorname{Li}_{1-k}\left((-1)^{2 / 3}\right)+4 i \pi^{k+1} \mathrm{Li}_{-k}\left((-1)^{2 / 3}\right)\right)$ \\
\hline$e^{m x} \operatorname{csch}(x)$ & $\pi\left(\tan \left(\frac{\pi m}{2}\right)-i\right)$ \\
\hline$\left(1-e^{m x}\right) \operatorname{csch}(x)$ & $-\pi \tan \left(\frac{\pi m}{2}\right)$ \\
\hline$x\left(1-e^{p x}\right) \operatorname{csch}(x)$ & $-\frac{1}{2} \pi^{2} \tan ^{2}\left(\frac{\pi p}{2}\right)$ \\
\hline$x^{3} \operatorname{csch}^{3}(x)$ & $-\frac{1}{8} \pi^{2}\left(\pi^{2}-12\right)$ \\
\hline$x^{4} \operatorname{csch}^{4}(x)$ & $-\frac{2}{45} \pi^{2}\left(\pi^{2}-15\right)$ \\
\hline$e^{u x} \operatorname{csch}(b x) \sinh (c x)$ & $\frac{\pi \sin \left(\frac{\pi c}{b}\right)}{b \cos \left(\frac{\pi c}{b}\right)+b \cos \left(\frac{\pi u}{b}\right)}$ \\
\hline$e^{u x} \operatorname{csch}(b x) \sinh (u x)$ & $\frac{\pi \tan \left(\frac{\pi u}{b}\right)}{2 b}$ \\
\hline$x e^{u x} \operatorname{csch}(b x) \cosh (c x)$ & $\frac{\pi^{2}\left(\cos \left(\frac{\pi c}{b}\right) \cos \left(\frac{\pi u}{b}\right)+1\right)}{b^{2}\left(\cos \left(\frac{\pi c}{b}\right)+\cos \left(\frac{\pi u}{b}\right)\right)^{2}}$ \\
\hline$\frac{e^{i b x}}{\sinh (\alpha)+\sinh (x)}$ & $\pi \operatorname{sech}(\alpha) \tanh \left(\frac{\pi b}{2}\right)\left(\operatorname{coth}^{2}\left(\frac{\pi b}{2}\right) \sin (\alpha b)+i \cos (\alpha b)\right)$ \\
\hline $\operatorname{csch}^{2}(x) \sinh ^{2}(m x)$ & $\frac{1}{2}(2-2 \pi m \cot (\pi m))$ \\
\hline$\frac{\operatorname{csch}(x)(\pi a \sin (m x)+x \cos (m x))}{\pi^{2} a^{2}+x^{2}}$ & $\frac{1}{a}-2 e^{\pi(-m)} \Phi\left(-e^{-m \pi}, 1, a+1\right)$ \\
\hline$\frac{\pi \operatorname{csch}(x)\left(\left(x^{2}-\pi^{2} a^{2}\right) \sin (m x)-2 \pi a x \cos (m x)\right)}{\left(\pi^{2} a^{2}+x^{2}\right)^{2}}$ & $2 e^{\pi(-m)} \Phi\left(-e^{-m \pi}, 2, a+1\right)-\frac{1}{a^{2}}$ \\
\hline
\end{tabular}

\section{Conclusions}

In this article the authors used their contour integral method to derive an Exponential Fourier transform in terms of the Lerch function. This transform was then used to produced a formal derivation for an Exponential Fourier transform listed in [8] and [4] along with providing the correct version for the integral in [4], with the aim of adding these results to current literature where such formulae are not listed. The Hilbert transform was also explored upon which new definite integrals were derived. We will be using our contour integral method to derive other integrals in our future work. 


\section{Acknowledgements}

We are very grateful to experts for their appropriate and constructive suggestions to improve this template.

\section{REFERENCES}

[1] Keith B Oldham; Jan C Myland; Jerome Spanier, An atlas of functions : with Equator, the atlas function calculator, New York, NY, Springer, 2009, English : 2nd ed, pp. 1-762.

[2] Abramowitz, M. and Stegun, I.A.(Eds), Handbook of Mathematical Functions with Formulas, Graphs, and Mathematical Tables, 9th printing, New York, Dover, (1982), pp. 1-1044.

[3] Bierens de Haan, D., Nouvelles Tables d'intégrales définies, Leide, P.Engels, Libraire Éditeur, Amsterdam, 1867, pp. 1-762.

[4] Erdéyli A. , Magnus W. , Oberhettinger F. , and Tricomi F.G. , Tables of Integral Transforms Vol. I, 1954, McGraw-Hill Book Company, Inc., New York-Toronto-London. pp. 1-410.

[5] Erdéyli A., Magnus W. , Oberhettinger F. , and Tricomi F.G. , Tables of Integral Transforms Vol. II, 1954, McGraw-Hill Book Company, Inc., New York-Toronto-London. pp.1-451.

[6] Finch, S.R., Encyclopedia of Mathematics and its Applications: Mathematical Constants, Cambridge, England, Cambridge University Press, (2003). pp. 1-618.

[7] Gradshteyn I.S \& Ryzhik I.M, Tables of Integrals, Series and Products, 6 Ed, Academic Press (2000), USA, pp. 1-1220.

[8] Prudnikov, A.P., Brychkov, Yu. A., Marichev, O.I. Integrals and Series, Elementary Functions, Volume. 1, USSR Academy of Sciences, Moscow, Taylor and Francis, (1986), pp. 1-802.

[9] Reynolds, R.; Stauffer, A. A Method for Evaluating Definite Integrals in Terms of Special Functions with Examples. 2020, International Mathematical Forum, Vol. 15, 2020, no. 5, 235-244.

https://doi.org/10.12988/imf.2020.91272

[10] Ametani A., Imanishi K., Development of exponential Fourier transform and its application to electrical transients Proceedings of the Institution of Electrical Engineers, Volume 126, Issue 1, pp. 51 -56

[11] Chinwuba IKE Charles, Exponential Fourier integral transform method for stress analysis of boundary load on soil ANNALS of Faculty Engineering Hunedoara-International Journal of Engineering Tome XVI, 2018, pp. 1-7. 